\title{
Non-destructive test of steel structures by conical indentation
}

\author{
Alexey Beskopylny ${ }^{1, *}$, Andrey Veremeenko ${ }^{1}$, Elena Kadomtseva $^{1}$, and Natalia Beskopylnaia ${ }^{1}$ \\ ${ }^{1}$ Don State Technical University, sq. Gagarin, 1, Rostov-on-Don, Russia
}

\begin{abstract}
The problem of mechanical properties measurement of steel structures by non-destructive method of impact cone indentation is considered. The method is based on analytical solution and experimental data of a problem of impact indentation by cone into elastic-plastic half-space. Nondestructive dynamic indentation method is one of more efficient for mechanical properties assessment because of compact instrument that makes it possible of measuring at any point of structure. The scheme of impact indentation was considered and the problem of elastic-plastic impact is solved. The device with a computer way of information processing was created and tested for determining the characteristics of the existing structures. The method was adopted and tested on bridge structures, butt welded joints, civil engineering structures and others.
\end{abstract}

\section{Introduction}

Organization of all types of non-destructive quality control is associated with the measurement of mechanical properties of structural elements and machine parts. However, currently, these properties can be determined only indirectly through the manufacture and special samples testing.

During manufacture structural elements are treated by complex types thermal, mechanical, chemical or another power, after which the material properties change. The most important mechanical properties are yield strength, tensile strength, elongation, Charpy impact strength and hardness. It needs to know these characteristics at all stages of the life cycle structure $[1,2]$. Resent researches $[3,4,5]$ show that characteristics of conical indentation allow us evaluate creep parameters and fracture toughness for some materials.

The shape of the indenter plays an important role in mechanical characteristics determining and specifies the algorithm test $[6,7]$. In practice widly known indenters in the form of a sphere [8,9], cone [10,11], pyramid, flat punch [12]. A large amount of testing of materials in industry poses the problem of the real structure testing instead of laboratory standart method. The destruction of most machine parts and structural elements is dynamic in nature or is a consequence of the dynamic application of the load. The complexity of the processes occurring during rapid load application resulted in a large number of methods for impact testing of materials in industry.

Quite an attractive scheme of testing the hardness when the indenter approaches the measured surface with an initial velocity $V_{0}$, the measure of the ratio of the speeds of approach and rebound. In this scheme used a number of indenters: spherical, conical, pyramidal. Studies of German companies, producing devices for measuring the hardness of materials, it is shown that such a scheme of hardness test is the most stable.

However, such methods require a high degree of preparation of the surface to be measured and can lead to indentors failure.

In this article, another test scheme is considered in which the hammer flies up to in the indenter rested on the surface to be measured. Dynamic measurement methods, in our opinion, are preferred because they allow to develop compact and convenient devices that could be used at any point of real structures.

\section{Problem definition}

It is known that the strain velocity during the test can significantly change the mechanical properties of the material. Therefore, in our case, it is advisable to use a range of speeds to $3 \mathrm{~m} / \mathrm{s}$. In that velocity range wave processes and inertial forces do not have a significant effect on the material reaction and can be neglected. So quasistatic models of stroke is applicable.

The shape of the indenter plays important role in the tests. For example during indentation of spherical indenters their prints are not geometrically similar for wide range of material properties. Conical and pyramidal indenters do not have these disadvantages because they give geometrically similar prints in the entire range of indentation depths.

We consider dynamic scheme (Figure 1) in which the striker impacts the initially immovable conical indenter with angle $\gamma$. The speed of the indenter in this case changes from zero to some maximum value $V_{\max }$, then falls to zero and changes sign during the rebound. This scheme is softer in comparison with the flying indenter and in this case the strain velocity will have less

\footnotetext{
Corresponding author: besk-an@yandex.ru
} 
effect on the change in mechanical properties, which can be effectively used in measurements.

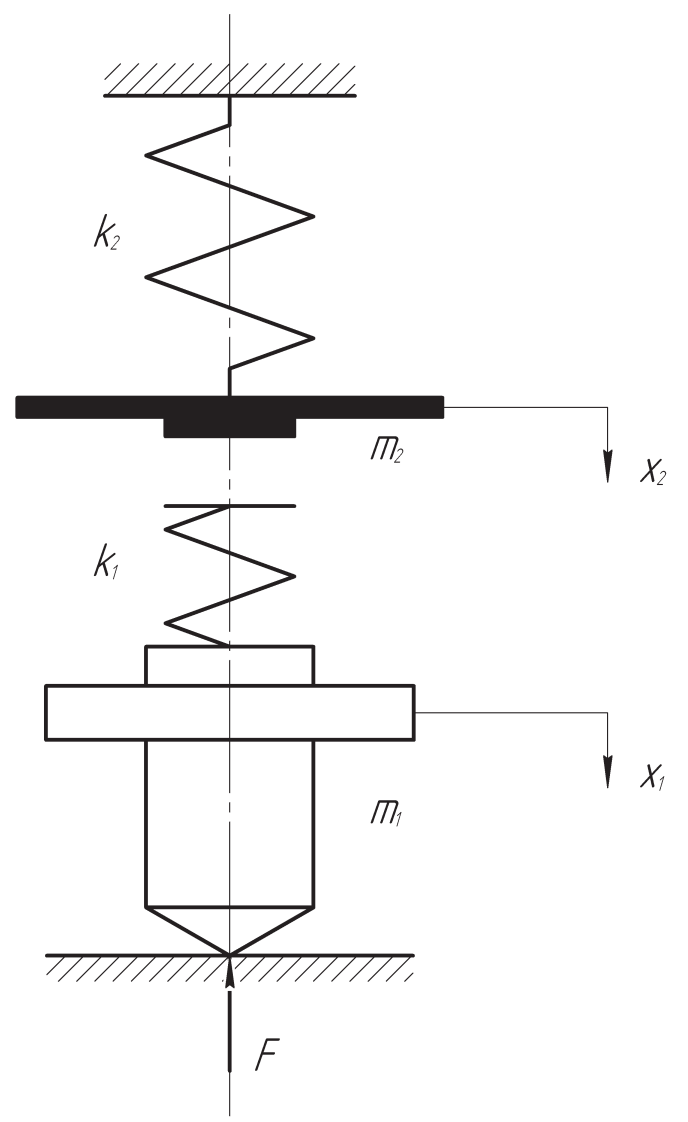

Fig. 1. Mechanical model of impact conical indentation.

The motion of the elements of the system is described by equations

$$
\begin{gathered}
m_{1} \ddot{x}_{1}=k_{1}\left(x_{2}-x_{1}\right)-F(t) \\
m_{2} \ddot{x}_{2}=k_{2}\left(x_{s t}-x_{2}\right)-k_{1}\left(x_{2}-x_{1}\right) \\
\text { at } x_{1}=0, x_{2}=0, \dot{x}_{1}=0, \dot{x}_{2}=V_{0}, x_{s t}=\text { const } .
\end{gathered}
$$

The problem of indentation of an elastic cone in elastic-plastic half-space by force $F$ that changes with time (Figure 2) is considered. We consider the cylindrical coordinates system; $\mathrm{z}$-axis is directed to coincide with the axis of the cone and facing down (inside the half-space). In the contact area $\Omega_{1}(\alpha)$ of cone with a half-space the friction is neglected, outside the area of contact $\Omega_{2}(\alpha)$ stresses $\sigma_{z r}$ and $\sigma_{z z}$ equate to zero. Plastic deformation begins under the Tresca conditions, the material at the same time characterizes the plastic constant $k=0,5 \sigma_{\mathrm{y}}$, where $\sigma_{\mathrm{y}}$ is yield strength, and indenter material undergoes only elastic deformation.

The displacement $u$ of any point of interacting solids is the sum of the elastic $u_{e}$ and plastic $u_{p}$ components:

$$
u=u_{e}+u_{p}
$$

The distribution of pressure in the contact zone has the form elastic solutions for any values of the force $F$

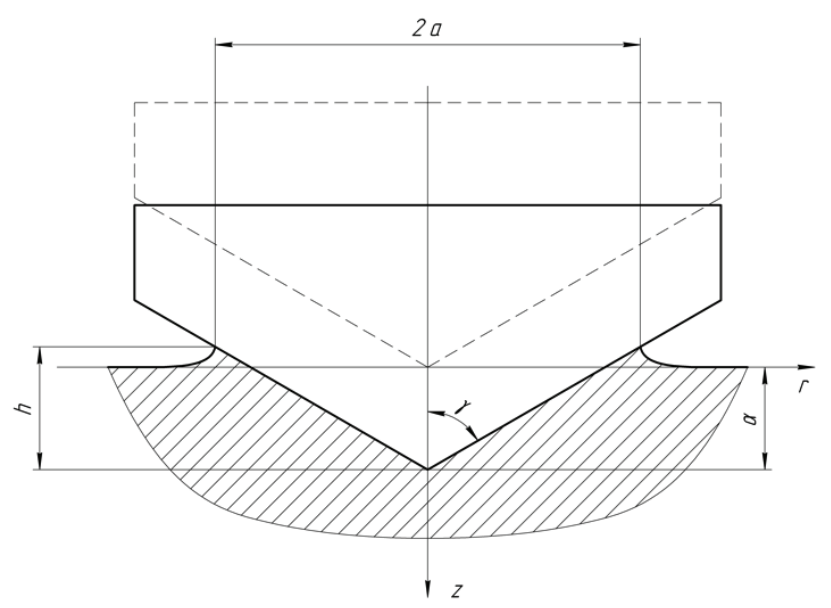

Fig. 2. The model of conical indentation.

$$
q(F, r)=-q_{0} \ln \left(a / r-\sqrt{(a / r)^{2}-1}\right),
$$

where $q_{0}=P /\left(\pi a^{2}\right)$ is the average pressure, $a$ - the contact area radius.

The increment of plastic deformations occurs when the conditions are performed

$$
q_{0}=\lambda k ; \quad d F / d t>0
$$

where $\lambda=5.7,2 k=\sigma_{\mathrm{y}}, \sigma_{\mathrm{y}}$ is material yield strength.

The average contact pressure does not exceed

$$
q_{0} \leq \lambda k
$$

Full indenter displacement

$$
\alpha=\alpha_{e}+\alpha_{p}=h_{p}\left(1-\delta^{*}\right)+\alpha_{e},
$$

where $h_{p}$ - depth of the residual print, taking into account the height of the inflow,

$\delta^{*}$ - coefficient determined at experiment.

So the dependence of indentation force $F(\alpha)$ is

$$
\alpha=C F^{1 / 2}
$$

$C=\operatorname{ctg}(\gamma)\left(1-\delta^{*}\right)(\lambda k \pi)^{-1 / 2}+\left(1+\left(2 \delta^{*}-2\right) / \pi\right)(\lambda k \pi)^{1 / 2} E^{-1}$.

Substituting the expression for $F(\alpha)$ (6) in equation (1) we get the dependence of the depth $S(t)$, the velocity $V(t)$ and acceleration $W(t)$ and contact force $F(t)$ during impact. The resulting equation contains material yield strength $\sigma_{\mathrm{y}}$ that can be determined during test. Other mechanical properties, such as tensile strength, elongation and impact strength, are closely related to the three parameters of the indentation: the depth of indentation $S=\alpha$, speed $V$ and acceleration $W$. 


\section{Results and discussion}

The theoretical and experimental studies made it possible to design of an instrument [13] for determining the mechanical properties of steels (Figure 3), which consist of handle 1 , spring 2 , striker 3 , body 4 , indenter holder 5 , sensor 6 and 9 , body part 7 , damper 8 .

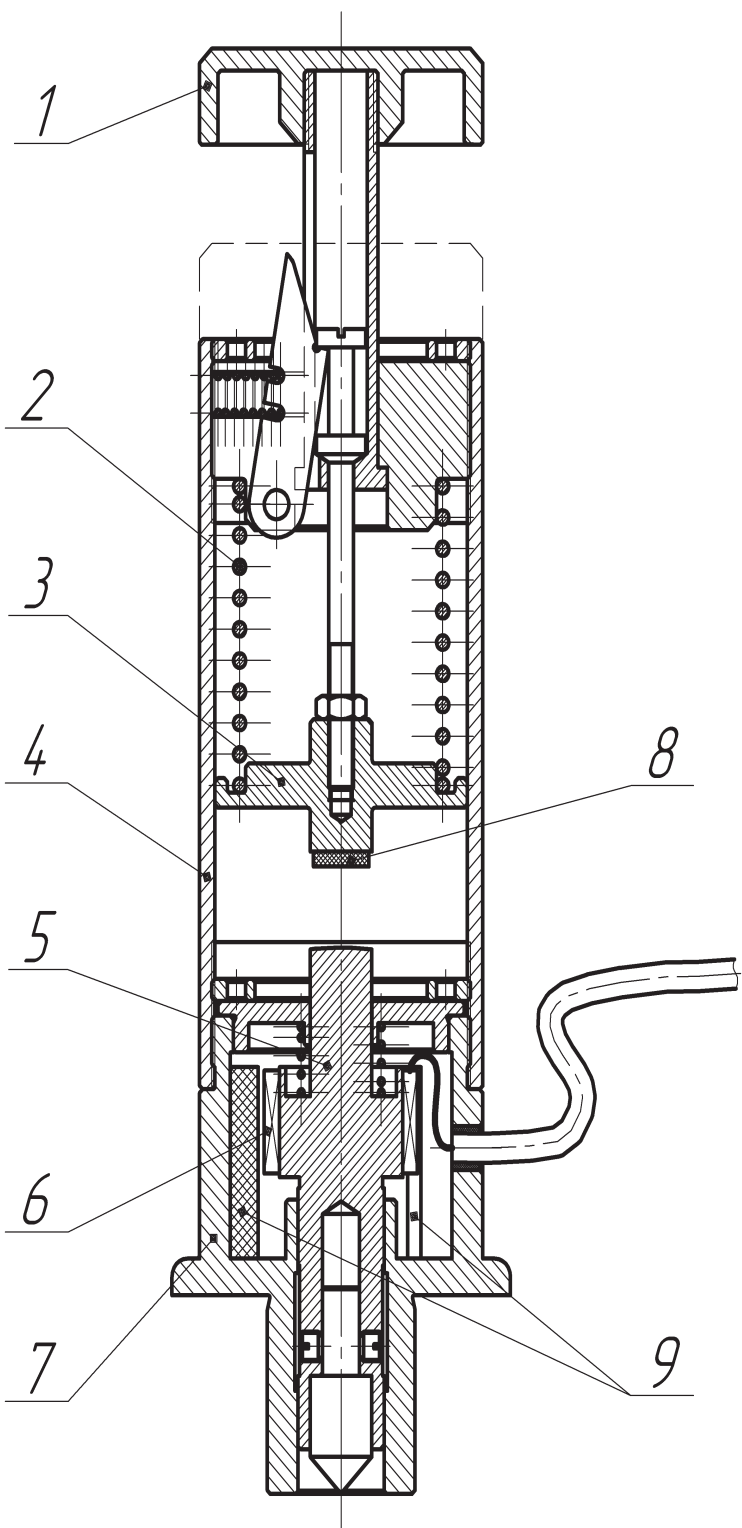

Fig.3. The devise for mechanical properties measurement.

During measurement striker 3 hits over the indenter holder 5 and sensor 6 registers signal of velocity $V(t)$. The dependences $S(t)$ and $W(t)$ are obtained by integrating and differentiating.

Figure 4 shows dependences of the depth $S(t)$, velocity $V(t)$, and acceleration $W(t)$ during impact indentation of a cone $90^{\circ}$ for two kinds of steel $100 \mathrm{HB}$ and $370 \mathrm{HB}$. The registration of the maximum and minimum values on these graphs makes it possible to determine the number of mechanical characteristics of steel.

The comparison of the obtained models with the experimental data was carried out on various structural steels in a wide range of mechanical characteristics. The proposed method makes it possible to estimate the yield strength with an error of $7 \%$, the tensile strength with an error of $5 \%$, the elongation of $10 \%$, and the toughness of $15 \%$.

\section{$\mathrm{S}, \mathrm{mm}$}



$\mathrm{V}, \mathrm{m} / \mathrm{s}$



$\mathrm{W},{ }^{*} 10^{3} \mathrm{~m} / \mathrm{s}^{2}$

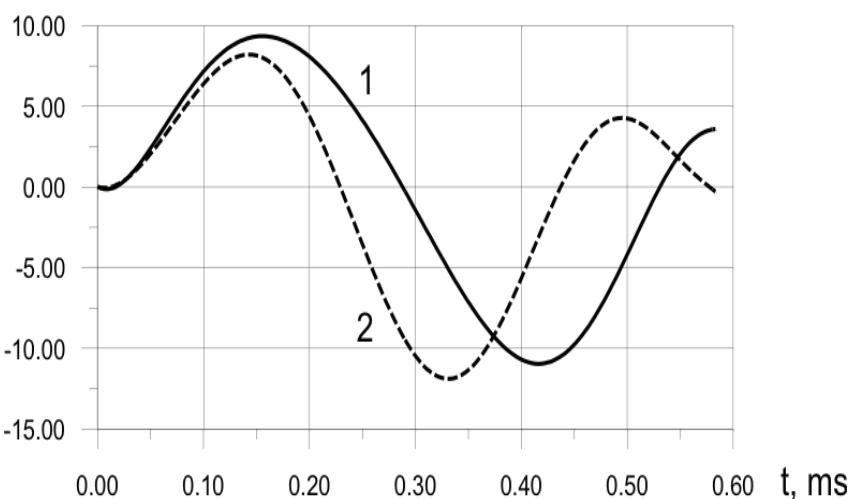

Fig.4. Dependences of the depth $S(t)$, velocity $V(t)$, and acceleration $W(t)$ during impact indentation of a cone $90^{\circ} .1$ steel $100 \mathrm{HB}, 2$ - steel $370 \mathrm{HB}$.

Subsequent computer processing of the signal is as follows. The original function of the indenter velocity $V(t)$ is defined on $[0, \mathrm{~T}]$ into $\mathrm{n}$ nodal points. The function is approximated by a cubic spline on each segment $\mathrm{t} \in\left[\mathrm{t}_{\mathrm{i}}, \mathrm{t}_{\mathrm{i}+1}\right]$ 


$$
\begin{gathered}
V(t)=y_{i+1} \tau+(1-\tau) y_{i}+ \\
+h\left[\left(M_{i}-\Delta y_{i}^{*}\right) \tau(1-\tau)^{2}-\left(M_{i+1}-\Delta y_{i}^{*}\right) \tau^{2}(1-\tau)\right] \\
\tau=\left(t-t_{i}\right) / h ; \quad \Delta y_{i}^{*}=\left(y_{i+1}-y_{i}\right) / h ; h=t_{i+1}-t_{i}
\end{gathered}
$$

Quadrature integration of the spline functions leads to simple expressions for displacement $S(t)$

$$
S(t)=\frac{h}{2} \sum_{i=0}^{n-1}\left(y_{i+1}+y_{i}+\frac{h}{6}\left(M_{i}-M_{i+1}\right)\right)
$$

To construct the acceleration curve $\mathrm{W}(\mathrm{t})$ the original function of the velocity $\mathrm{V}(\mathrm{t})$ was approximated by a polynomial of the 9th degree. Differentiation of this polynomial gives a function of acceleration $W(t)$ at a given period of time $\mathrm{t} \in[0, \mathrm{~T}]$

\section{Conclusion}

Existing methods for determining the mechanical characteristics of steels do not allow measurements directly on the structure in operating conditions with a high degree of accuracy. Developing a method and a series of portable instruments that allow controlling mechanical properties by non-destructive test can solve such a problem.

On the basis of analytical, design and metrological developments, new portable impact devices for determining the mechanical properties of steels have been created and their accuracy has been estimated.

The impact energy of the striker is $0.16 \mathrm{~J}$, which corresponds to the work done by the force of indentation on the Rockwell instrument for a material with a hardness of 45 HRC. For the hit device scheme shown in Fig. 1, the energy of impact is determined by the formula

$$
E=\frac{k_{2}}{2} z_{0}^{2}\left[\sin \left(\arccos \frac{z}{z_{0}}\right)\right]^{2}
$$

where $z_{0}$ is the preliminary compression of the spring, m;

$z$ - coordinate of the holder of the indenter relative to the equilibrium position of the spring, $\mathrm{m}$;

$k_{2}$ - rigidity of the main trigger spring, $\mathrm{N} / \mathrm{m} ; k_{2}=$ $500 \mathrm{~N} / \mathrm{m}$.

The model of the device with a computer way of information processing was tested in determining the characteristics of the existing structures [14], butt welded joints [15] and others.

\section{References}

1. D.M. Belenkyi, A.N. Beskopylnyi, N.L. Vernesi, News of higher educational institutions. Construction 1, 99-102 (2003)

2. A.N. Beskopylny, I.G. Kadomtsev, N.I. Beskopylnaya, Theoretical foundations of civil engineering, 83-84 (2005)

3. A.S. Chepurnenko, V.I. Andreev, B.M. Yazyev, A.N. Beskopylny, MATEC Web of Conferences 67, 06059 (2016)

4. S. Chatterjee, Sanjay Panwar, K. Madhusoodanan, A. Rama Rao, Nuclear Engineering and Design 305, 9-17 (2016)

5. J. Dean, J. Campbell, G. Aldrich-Smith, T.W. Clyne, Acta Materialia 80, 56-66 (2014)

6. A.R.H. Midawi, C.H.M. Simha, M.A. Gesing, A.P. Gerlich, International Journal of Solids and Structures 104-105, 81-91 (2017)

7. A.N. Beskopylnyi, A.A. Lyapin, Recent trend in Science and Technology management 23-29, 36-45 (2016)

8. J. Dean, T.W. Clyne, Mechanics of Materials 105, 112-122 (2017)

9. A.S. Useinov, K.S. Kravchuk, A.A. Rusakov, I.V. Krasnogorov, A.P. Kuznetsov, T.V. Kazieva, Physics Procedia 72, 194-198 (2015)

10. I. Mohagheghian, W.J. Stronge, G.J. McShane, European Journal of Mechanics 61, 134-150 (2017)

11. I.I. Argatov, International Journal of Solids and Structures 48, 3444-3452 (2011)

12. Wei-Min Chen, Yang-Tse Cheng, Min Li, Materials Science and Engineering 527, 5613-5618 (2010)

13. D.M. Belenkii, A.N. Beskopylnyi, N.N. Beskopylnyi, E.K. Polibin, B.A. Pesenko Method for determining mechanical characteristics and the device for its implementation. Patent No. 2079831 (1997)

14. D.M. Belenkii, A.N. Beskopylnyi, N.L. Vernezi News of Higher Education Institution. Construction. 1, 99-102 (2003)

15. D.M. Belenkii, A.N. Beskopylnyi, N.L. Vernezi, L.G. Shamraev Welding International, №11 (8), pp. 642-645 (1997) 